\title{
Atmospheric aerosol measurements from satellites
}

\author{
Nataliia Borodai ${ }^{1, \star}$ \\ ${ }^{1}$ Institute of Nuclear Physics Polish Academy of Sciences, PL-31342 Krakow, Poland
}

\begin{abstract}
Satellite instruments are commonly used for measurements of aerosol optical properties in the atmosphere. In this work we use such measurements for ultra-high energy cosmic ray studies. We introduce a method of preparation of aerosol maps for the location of the Pierre Auger Observatory. For these maps the data from the Ozone Monitoring Instrument (OMI) of the Aura satellite were analyzed for the period 2004-2016. Such aerosol properties as the aerosol optical depth (AOD) and aerosol types were retrieved. The seasonal dependence of aerosol distribution is also studied.
\end{abstract}

\section{Introduction}

Aerosols affect the radiation budget of the Earth's atmosphere directly by scattering and absorbing solar radiation. They also influence indirectly clouds, modifying their optical properties and lifetime. Aerosols in the atmosphere influence the detection of extensive air showers developing in the atmosphere. This is why it is important for the cosmic ray physics to know the distribution of aerosols in the atmosphere.

Currently there are several instruments for aerosol measurements used at the Pierre Auger Observatory [1],[2]. Among them are Central Laser Facility (CLF) [3] and eXtreme Laser Facility (XLF), LIDAR stations located near each fluorescence detector building [4], and others. However, these instruments do not give the global picture of aerosols over the Observatory. They measure aerosols only along a certain direction. Measurements done using satellite data allow one to produce aerosol maps, which show the periodic increases and decreases of aerosols in time over the whole Observatory. This is especially important since the fluorescence detectors of the Observatory view the sky over large areas and the aerosol concentrations may vary at different locations within their field of view.

There are a number of satellites with instruments capable of measuring aerosols in the Earth's atmosphere. Among these instruments is the Ozone Monitoring Instrument (OMI) [5] aboard NASA's Earth Observing System's (EOS) Aura satellite. Aura was launched on July 15, 2004, and OMI has collected data since August 9, 2004.

The Aura spacecraft is in a polar sun-synchronous orbit at an altitude of $705 \mathrm{~km}$ and has a daily global coverage with on-average 14 orbits. Aura flies in a formation of satellites, called "A-Train" (the Afternoon constellation) [6]. The primary goal of the constellation is to provide ob-

\footnotetext{
^e-mail: nataliia.borodai@ifj.edu.pl
}

servations of clouds and aerosols which are not possible from ground-based instruments.

\section{OMI aerosol measurements}

The OMI is a nadir-viewing spectrometer. It is a kind of passive imager which measures the amount of solar radiation backscattered by the Earth's atmosphere and surface.

In this study we used reflected solar radiation measurements from the OMI instrument to estimate the aerosol optical depth (AOD) over the Pierre Auger Observatory. The AOD is the aerosol extinction coefficient integrated vertically from the surface to the top of the atmosphere.

\subsection{OMI instrument}

The OMI instrument measures the solar backscattered radiance in the wavelength range between 270 and $500 \mathrm{~nm}$, with a spectral resolution between $0.42 \mathrm{~nm}$ and $0.63 \mathrm{~nm}$. In this wavelength range, the scattering and absorption by atmospheric aerosol particles varies smoothly with wavelength. The wavelength dependence is used to obtain information on the aerosol type, concentration and the size distribution. The nadir pointing telescope of OMI has a very large field of view of $114^{\circ}$, which is used for swath registration, perpendicular to the flight direction of the satellite. Due to so large a field of view, the OMI swath width is $2600 \mathrm{~km}$, which provides daily global coverage. A nominal ground footprint or ground pixel size is $13 \times 24$ $\mathrm{km}^{2}$ at nadir.

Figure 1 represents the real OMI measurements done over the location of the Pierre Auger Observatory on June 9, 2013. Green points represent ground pixel centers, numbers show the sequence of measurements (every 2 sec).

The time of the measurements over the Pierre Auger Observatory is usually the evening time: around 17.0019.00 UTC (14.00-16.00 local time). 
Longitude/Latitude, June 9

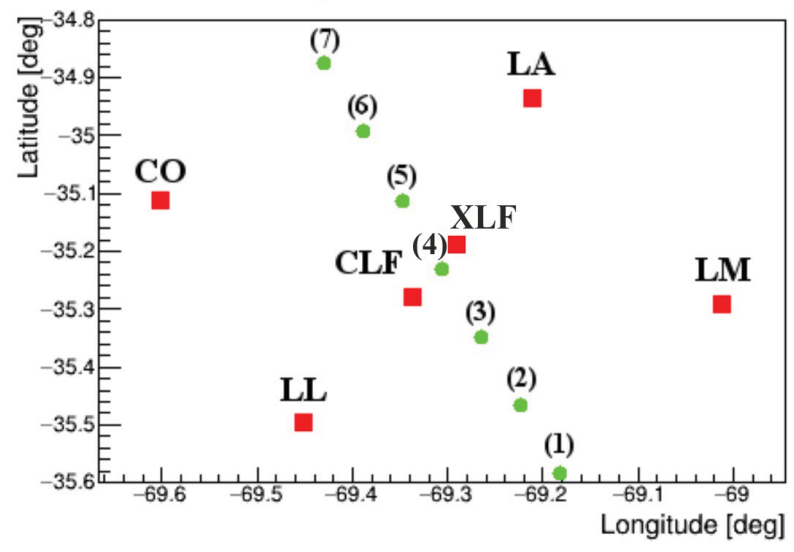

Figure 1. The OMI measurements over the Pierre Auger Observatory performed on June 9, 2013. Red squares correspond to locations of the fluorescence detectors (LA, LM, LL, CO) [2] and the laser facilities (CLF and XLF) at the Pierre Auger Observatory. Green points represent ground pixel centers, the numbers show the sequence of measurements.

Among OMI capabilities are a measurement of aerosol optical depth (AOD), the ability to distinguish between aerosol types, such as smoke, dust and sulfates, the ability to measure aerosol absorption capacity in terms of aerosol absorption optical depth or single scattering albedo. The AOD and aerosol type determinations are the main properties studied in this work to be used in cosmic ray shower investigations.

\subsection{Retrieval algorithms and aerosol types}

There are two algorithms [7] used for aerosol retrieval: Near UV aerosol retrieval algorithm (OMAERUV) and Multi-wavelength Algorithm (OMAERO).

OMAERUV is used to retrieve the AOD values over land. It uses as input OMI measured reflectances at 354 and $388 \mathrm{~nm}$ to retrieve column atmosphere values of AOD, using a set of 21 aerosol models. The algorithm differentiates the types of aerosols: desert dust, biomass burning carbonaceous particles, sulfate-based aerosols.

OMAERO is used to retrieve the AOD values preferably over ocean. It uses the measurements from 15 wavelengths in the range from 342.5 to $483.5 \mathrm{~nm}$.

In the following we use only the data retrieved using the OMAERUV algorithm as it is more common for over land measurements.

There are three aerosol types distinguished in OMI data in the OMAERUV algorithm: smoke (biomass burning carbonaceous particles), desert dust and sulfate-based aerosols. Taking all the available data for the location of the Pierre Auger Observatory for the years 2004-2016 and applying some cuts (see cuts 1-3 described in section 2.3) Figure 2 was produced. This figure shows the number of satellite measurements of aerosols classified according to their types defined from the OMAERUV algorithm. The number of measurements is plotted vs month to see the seasonal changes of the quantities of aerosols of different

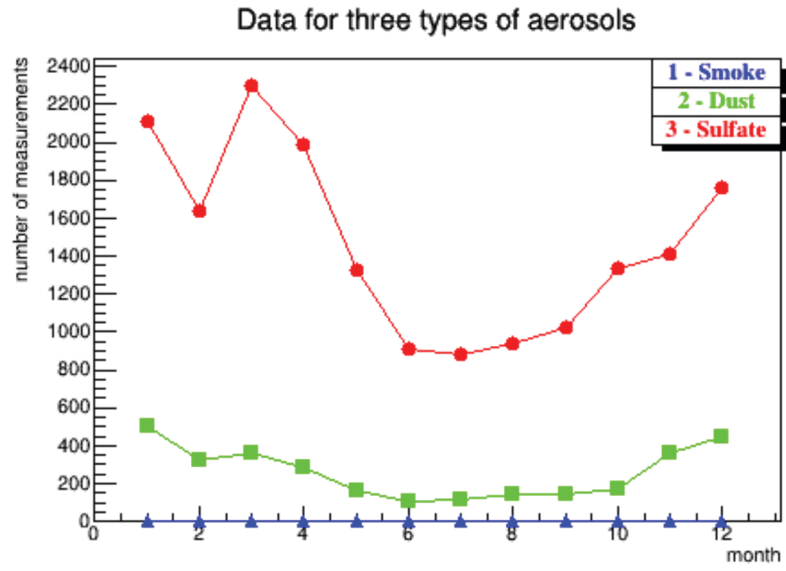

Figure 2. Number of OMI measurements over the Pierre Auger Observatory over years 2004-2016 estimated for three types of aerosols. Blue triangles represent smoke, green squares are dust and red dots are sulfate-based aerosols.

types. It appears that sulfate-based aerosols constitute the main part of the aerosols (more than $85 \%$ ).

According to OMI models the distribution of the sulfate-based aerosols decreases exponentially with height. Other aerosol types have distributions with maximum at some altitude. So, in the following only sulfatebased aerosols are taken into account for AOD estimation.

\subsection{Dataset}

The OMAERUV algorithm data with wavelength $354 \mathrm{~nm}$ are chosen for the following analysis. This wavelength is very close to the one used by the CLF instrument at the Pierre Auger Observatory (355 nm).

There are some standard satellite data quality flags [8], such as: row anomaly correction flag, pixel quality flags, measurement quality flags, algorithm flag. These quality selection criteria should be applied to obtain the OMI level 2 data, which are used in the current analysis.

In summary, to obtain a more clean dataset the following cuts on data are applied: 1. OMAERUV algorithm AOD values are chosen; 2 . the data with the wavelength $354 \mathrm{~nm}$ are taken; 3 . satellite data quality flags are applied; 4. only sulfate-based aerosols are taken for AOD estimation.

In this work the satellite dataset is limited to the temporally averaged data. So, after applying the cuts all available OMI measurements were taken for the period from $\mathrm{Au}-$ gust 2004 to June 2016 for the region of the Pierre Auger Observatory. Each available data point corresponds to the ground pixel with its own longitude and latitude coordinates (same as shown in Figure 1). All these data points were then mapped according to their positions. The map with the points was then gridded at $0.2^{\circ} \times 0.2^{\circ}$ to build the aerosol map. Finally, the map was plotted as a $2 \mathrm{D}$ histogram smoothed with cell size of about $0.04^{\circ} \mathrm{x} 0.04^{\circ}$ as shown in Figure 3. The mean $<\mathrm{AOD}>$ is provided along with its RMS, standard deviation of the mean and the number of the data points. 

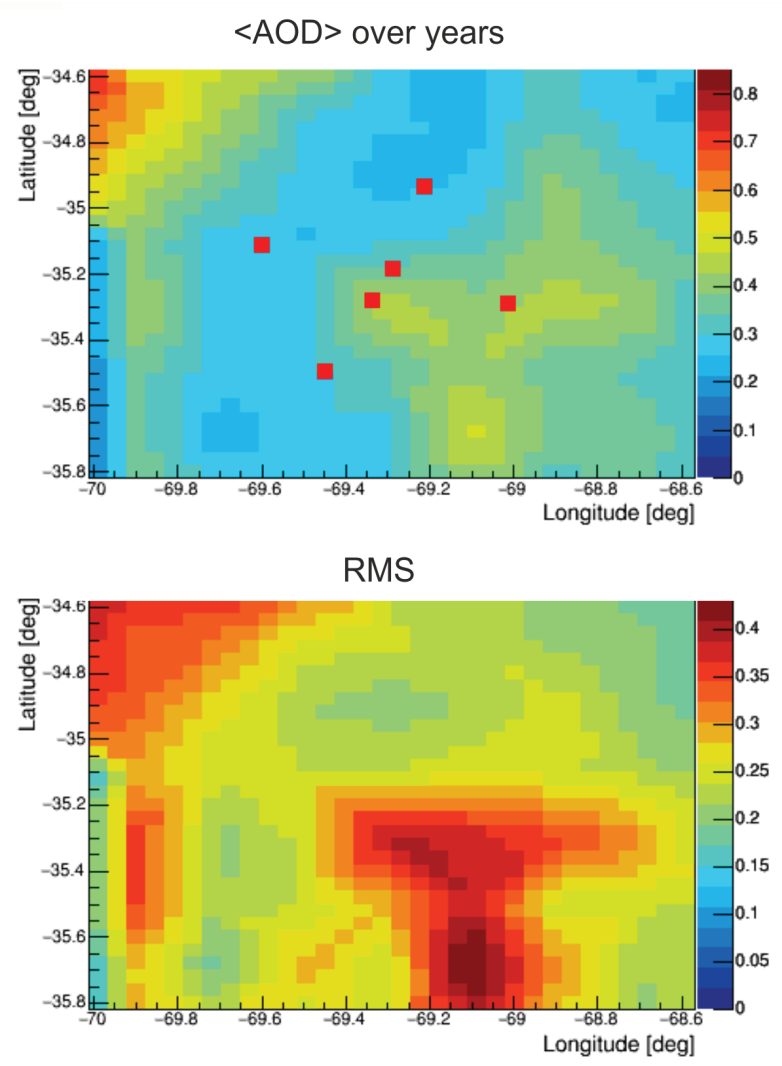

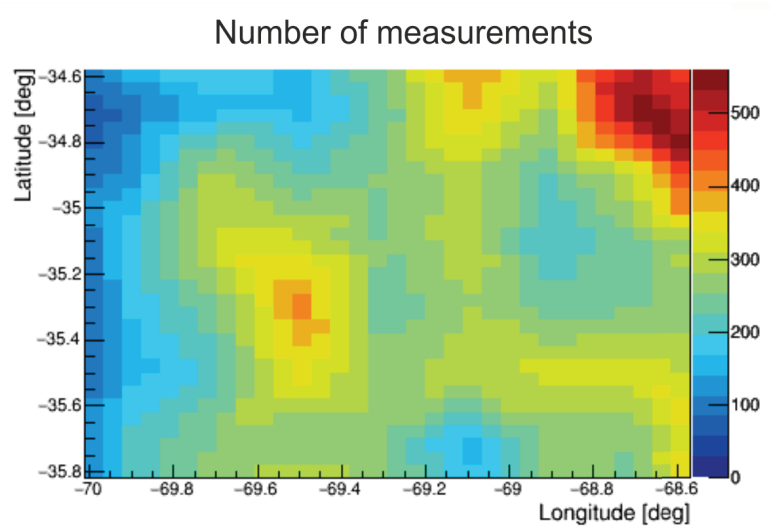

Standard deviation of the mean <AOD>

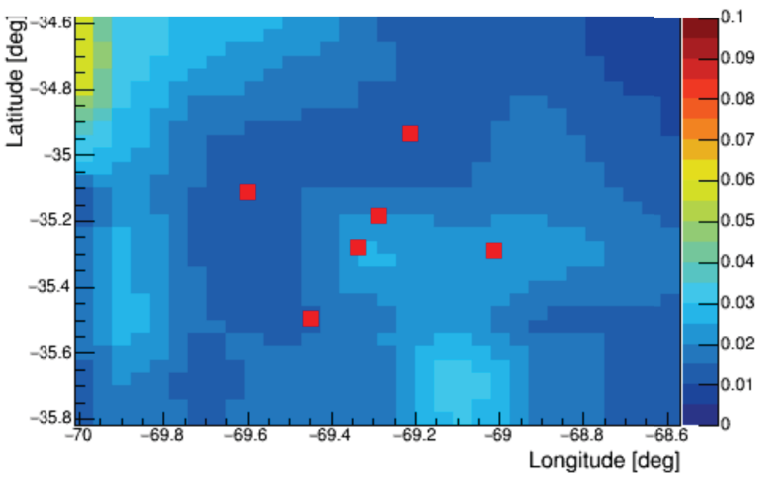

Figure 3. Estimation of $\langle A O D>$ and its errors for years 2004-2016 for the region of the Pierre Auger Observatory. Red squares represent the fluorescence detectors and the lasers (as shown in Figure 1). Top-left image: average <AOD> map; top-right image: number of AOD measurements averaged over years; bottom-left image: estimated RMS for $<\mathrm{AOD}>$; bottom-right image: estimated standard deviation of the mean $<\mathrm{AOD}>$.

\section{Results and discussion}

According to the procedure described in section 2.3 the aerosol maps over the Observatory were produced. Average values of $<\mathrm{AOD}>$ (top-left image in Figure 3) may suggest that aerosols are inhomogeneous over the Pierre Auger Observatory.

It should be mentioned that the OMI measurements of AOD are not confirmed by the aerosol measurements performed at the Pierre Auger Observatory using CLF (viewed from each of the FD sites), which point out that the aerosols are close to being homogeneous [1]. In addition, $<$ AOD $>$ values obtained from satellite are up to several times larger than the values measured at the Pierre Auger Observatory, and so are inconsistent with the ground-based measurements.

One should keep in mind that OMI data analysis relies heavily on models to describe the state of the atmosphere. A valid question is whether the global atmospheric models used in satellite data reduction describe well the local conditions at the site? It is well known that to correctly describe the real atmosphere it is necessary to account for relatively quick time variations of the state of the atmosphere [9].

A possible explanation for the large absolute values of satellite AOD values can be sub-pixel cloud contamination which was reported by OMI group [8]. It happens because of the relatively large pixel size of the OMI observations. So, the mean AOD can be significantly over- estimated. On the other hand, according to [8], the experience with the OMI predecessor satellite spectrometer TOMS (Total Ozone Mapping Spectrometer) suggests that monthly mean $<$ AOD $>$ values do reliably capture variation in the AOD with time.

One more source of uncertainty is the OMI cloud screening scheme, due to which the data with cloud days can be coincidentally included to the analysis. So, in the future analysis the OMI data should be used together with cloud information from other sources to exclude partly clouded OMI scenes that are not recognized as being cloudy by OMI.

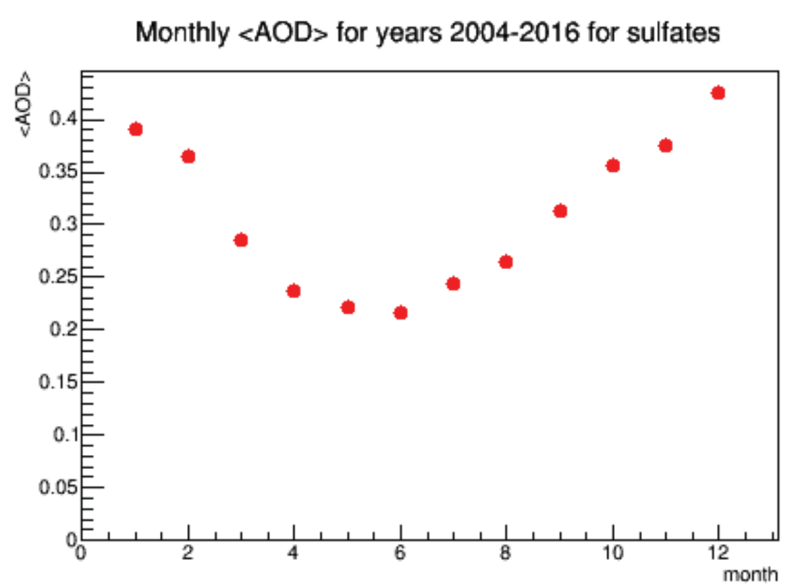

Figure 4. Monthly $<\mathrm{AOD}>$ for sulfates averaged for the years 2004-2016. 
$<$ AOD > map for summer months $(12,1,2)$

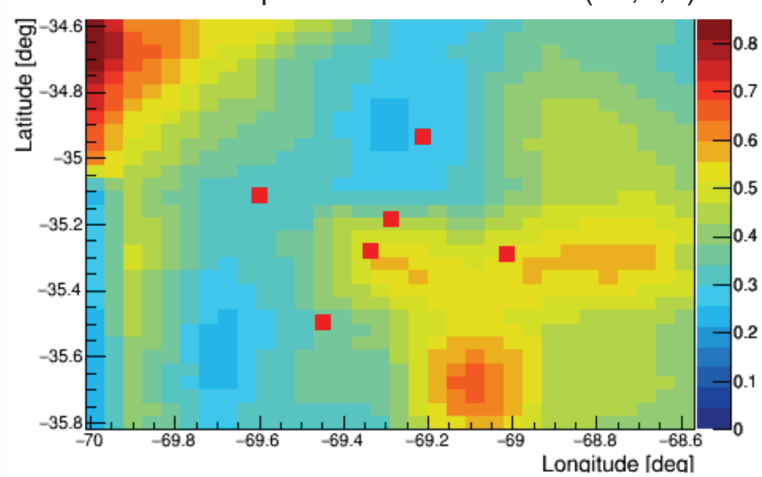

$<$ AOD $>$ map for winter months $(6,7,8)$

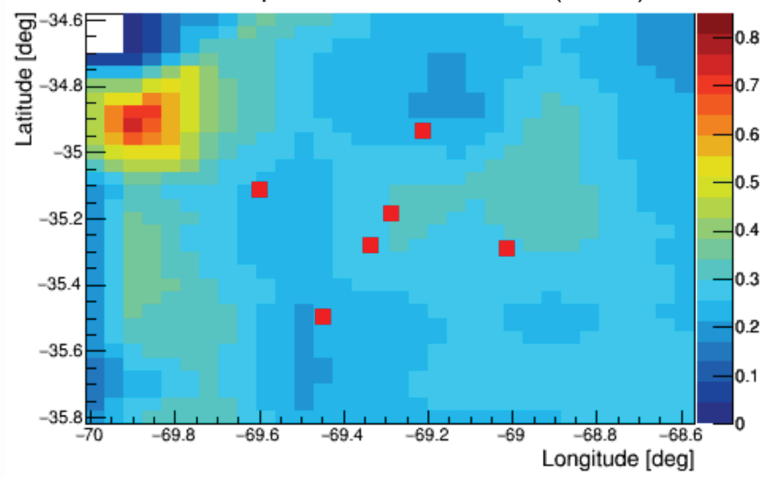

<AOD> map for autumn months $(3,4,5)$

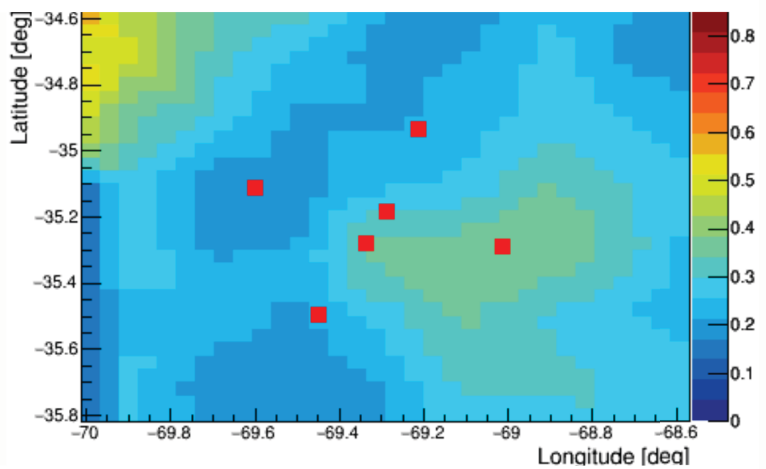

$<$ AOD $>$ map for spring months $(9,10,11)$

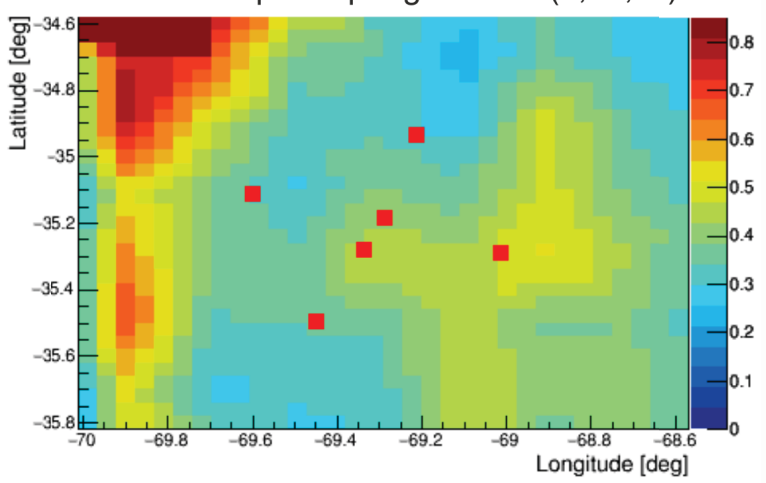

Figure 5. Seasonal $<$ AOD $>$ maps for years 2004-2016 produced for the location of the Pierre Auger Observatory.

However, although the average values of AOD from OMI seem to have problems, it may be interesting to look at seasonal variation of aerosols.

Using the described dataset the monthly average $<$ AOD $>$ was obtained for sulfates (see Figure 4).

In Figure 5 the seasonal changes of the aerosols are presented. These maps correspond to the results presented in Figure 4, showing the highest values of $<\mathrm{AOD}>$ during summer and the lowest during winter.

\section{Conclusion}

This work is an attempt to analyse aerosol data from polar satellites for the purposes of cosmic ray physics. The information obtained from this analysis may cause many questions. Although the OMI satellite-based aerosol measurements point out the inhomogeneity of aerosols over the Pierre Auger Observatory, the question about the existence of this inhomogeneity is still open as the quality of the OMI aerosol data is not sufficient to prove the obtained results. Among the questions there is also one about sulfates: why do we see such a large fraction of sulfate-based aerosol emissions in that region? Can they be due to a large content of volcanic ash in the soil? For the time being, we do not have answers to those questions.

So, further studies of $\angle$ AOD $>$ are definitely needed. It may be useful to use the cloud and aerosol information from other satellites. Particularly, satellites that fly in the A-Train formation together with Aura satellite possess the instruments for cloud and aerosol analysis, namely: the global imager MODIS on EOS-Aqua, the cloud radar on CLOUDSAT, the aerosol/cloud lidar on CALIPSO.

\section{Acknowledgments}

This work was partially supported by the National Science Centre under grant 2013/08/M/ST9/00728.

The author also thanks NASA's Goddard Earth Sciences Data Information Services Center (GES DISC) and the OMI aerosol research team for providing the satellite data used in this study.

\section{References}

[1] The Pierre Auger Collaboration, Astropart. Phys. 33, 108 (2010)

[2] The Pierre Auger Collaboration, Nucl. Instrum. Meth. A 798, 172 (2015)

[3] B. Fick et al., JINST 1, P11003 (2006)

[4] S. BenZvi et al., Nucl. Instrum. Meth. A 574, 171 (2007)

[5] OMI Algorithm Theoretical Basis Document, Volume 1 (ATBD-OMI-01), (2002)

[6] NASA Facts, A-Train Factsheet (Fs-2003-1-053gsfc), Formation Flying: The Afternoon "A-Train" Satellite Constellation (2003)

[7] OMI Algorithm Theoretical Basis Document, Volume 3 (ATBD-OMI-03), (2002)

[8] Ozone Monitoring Instrument (OMI) Data User's Guide (OMI-DUG-5.0), (2012)

[9] B.Wilczyńska et al., Astropart. Phys. 25, 106 (2006) 